\title{
Unilateral herpes zoster ophthalmicus with bilateral cerebral infarcts in human immunodeficiency virus seropositive patient
}

\author{
Girish Baburao Kulkarni, Veerendrakumar Mustare, Masoom Abbas Mirza \\ Department of Neurology, National Institute of Mental Health and Neurosciences, Bengaluru, Karnataka, India
}

\section{ABSTRACT}

Ischemic stroke is a recognized complication of herpes zoster ophthalmicus. Arterial involvement is usually seen on the side of the rash. It is thought to be due to vessel inflammation by the virus, which travels from the trigeminal ganglion. Few case reports of bilateral and distant site of zoster lesions with stroke in the brain have been described. These reports suggest possibility of contiguous vascular, cerebrospinal fluid (CSF) or hematogenous spread of the virus from the ganglion to the cerebral blood vessels. Therapeutically acyclovir, anticoagulation, and steroids have been used in the treatment of the zoster associated with stroke. We describe a case of immunocompromised patient with ipsilateral zoster ophthalmicus with bilateral anterior circulation strokes, who was treated with above measures and made successful recovery. This report also raises/supports possible CSF/vascular/hematogenous spread of the virus from the ganglion to involve cerebral blood vessels leading to the stroke.

Key words: Acyclovir, herpes zoster ophthalmicus, human immunodeficiency virus, stroke, varicella zoster virus

\section{Introduction}

Stroke is a known manifestation of human varicella zoster virus (VZV) infection seen in children with chicken pox and in adults with zoster. ${ }^{[1]}$ This VZV associated vasculopathy affects either large (granulomatous arteritis) or small vessels in both immunocompetent and compromised patients. It is thought that the virus travels from the ipsilateral trigeminal ganglion through its branches to the vessel wall and cause inflammation. There are also few reports that raise a possibility of contiguous vascular, cerebrospinal fluid (CSF), or hematogenous spread of the virus from the ganglion (Trigeminal/Thoracic) to the cerebral blood vessels. ${ }^{[2-5]} \mathrm{In}$ this case report, we describe an immunocompromised patient secondary to human immunodeficiency virus infection (HIV) with left herpes zoster ophthalmicus

\begin{tabular}{|l|l|}
\hline \multicolumn{2}{|c|}{ Access this article online } \\
\hline Quick Response Code: & Website: \\
\hline & www.ruralneuropractice.com \\
\cline { 2 - 3 } & \\
\hline & DOI: \\
\hline
\end{tabular}

who subsequently developed bilateral cerebral infarctions. He was successfully treated with acyclovir, methylprednisolone, and anticoagulation.

\section{Case Report}

A 43-year-old male was brought to our hospital by his elder sister with history of sudden onset of inability to understand the speech and bumping on to objects on the right side of his way and later on progressively becoming drowsy for duration of 15 days. There was no history of seizure, ear discharge, and fever. He was a known HIV seropositive patient not on any treatment and his CD4 count and viral load were not available. Three months prior to this illness, he had developed skin lesions suggestive of herpes zoster involving his left forehead and periorbital area's with redness of the eye. He was treated by an ophthalmologist with oral Acyclovir tablets, analgesics, and eye drops for 14 days. The lesions had healed during next 15 days with mild scarring of the left cornea with impaired vision. There was no previous history of hypertension, diabetes, and cardiac disease.

On examination he had the scar of herpes zoster with keratitis on the left side. Peripheral pulses, blood

Address for correspondence:

Dr. Girish Baburao Kulkarni, Associate Professor Department of Neurology, Hosur Road, Bangalore - 560 029, Karnataka, India.

E-mail: gbk5000@rediffmail.com 
pressure, and cardia were normal. Neurologically patient was drowsy but arousable, not responding to simple oral commands. He was not able to point to the objects. He was speaking some words unrelated to context of the conversation. Reading, writing, and repetition could not be tested. Pupils were $3 \mathrm{~mm}$ reactive to light, optic fundi were normal. Eye closure to the visual threat on the right side was absent. He had mild right upper motor neuron facial palsy. There was spasticity of all four limbs (right more than left) with mild right sided weakness requiring one-person support to walk.

Investigations revealed normal hemoglobin, erythrocyte sedimentation rate, vasculitis profile, renal and hepatic parameters, electrolytes, lipid profile, cardiac evaluation, and serum venereal disease research laboratory (VDRL) test. His HIV serology was positive with CD4 count of $191 / \mu 1$. Ultrasound Doppler of neck vessels was normal. Magnetic resonance imaging (MRI) of the brain plain and contrast [Figure 1] showed left temporal, left parietal, left basal frontal and periventricular and right basal ganglionic infarcts with hemorrhagic transformation. Magnetic resonance angiography (MRA) showed normal extracranial carotids and left vertebral artery. The distal left internal carotid, bilateral middle cerebral arteries were irregular with nonvisualization of the left anterior cerebral artery [Figure 2]. Right vertebral artery was hypoplastic. CSF analysis showed protein $-0.6 \mathrm{~g} / \mathrm{L}$, glucose $-0.63 \mathrm{~g} / \mathrm{L}$ with cell count -25 cells/ $\mu$ l (all lymphocytes). India ink, cryptococcal antigen, antitoxoplasma, antimycobacterial, and anticysticercal antibodies were negative. Cultures for bacterial and mycobacterium organisms were negative. Very high titers of CSF IgG antibodies to VZV (5000 IU/ml) by enzyme linked immuno absorbent assay (ELISA) were seen while IgM antibodies were

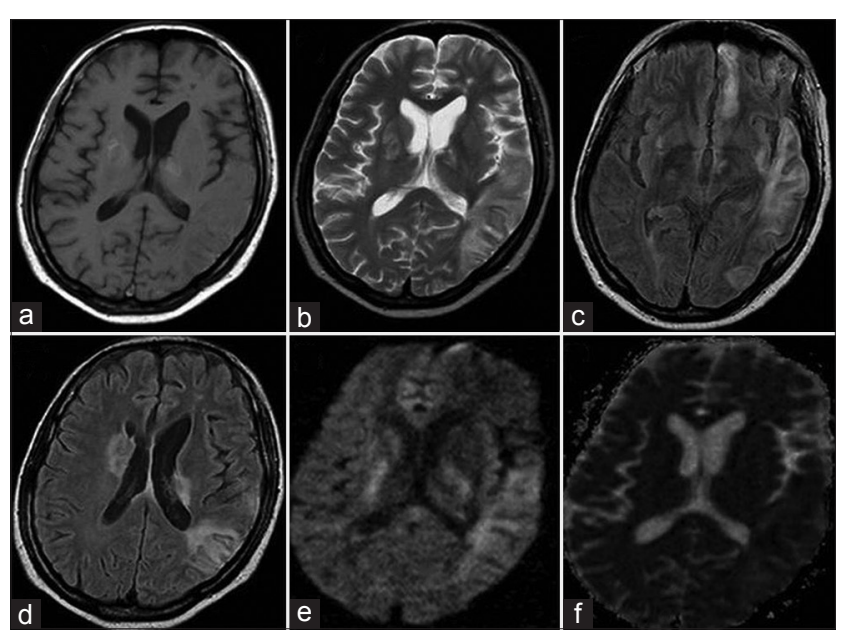

Figure 1: Magnetic resonance imaging of the brain T1 (a), T2 (b), Fluid attenuated inversion recovery ( $c$ and d), diffusion weighted image (e) and apparent diffusion weighted image (f) Sequences showing bilateral infarcts with hemorrhagic transformation negative. Electro encephalogram showed diffuse slowing of the background activity.

He was diagnosed as a case of left herpes zoster ophthalmicus with vasculopathy with acquired immuno deficiency syndrome (AIDS) with bilateral ischemic infarcts. He was treated with intravenous Acyclovir $500 \mathrm{mg}$ $8^{\text {th }}$ hourly for 14 days (weight: $48 \mathrm{~kg}$ ), methylprednisolone $1 \mathrm{~g} /$ day for 5 days, injection heparin followed by oral acenocoumarol with prothrombin time (international normalization ratio) maintained at around 1.5 for 3 months. He also received other supportive therapy during hospitalization and tab clopidogrel $75 \mathrm{mg} /$ day after 3 months. Over the next 15 days, the patient gradually improved in the sensorium and language function, in the form that he could understand simple commands, indicate his toilet needs, and walk independently. Repeat CSF examination done after 6 months was normal (cells $-3 / \mu \mathrm{l}$, protein $-0.25 \mathrm{~g} / \mathrm{L}$ repeat antibodies not done). He was also started on antiretroviral therapy and 2 years in to his follow-up he is maintaining good health with normalization of the CD4 counts $\left(700\right.$ cells $\left./ \mathrm{mm}^{3}\right)$.

\section{Discussion}

Our patient who was a known case of HIV seropositive state presented with bilateral cerebral infarcts 3 months after left zoster ophthalmicus. The internal, middle and anterior cerebral arteries ipsilateral to the zoster and branches of the contralateral middle cerebral arteries were involved in the form of irregularity and beading of the vessels. Other causes of stroke were excluded by appropriate history, examination, and investigations and it was attributed to varicella vasculopathy with positive CSF IgG anti VZV antibodies. Following treatment with acyclovir, short course steroid therapy, anticoagulants, and antiplatelets and later on with antiretroviral therapy he made significant recovery and is on follow-up.

The salient and interesting features about the present patient are (a) bilateral involvement of the cerebral vessels with unilateral zoster ophthalmicus with immunocompromised state and (b) favorable response

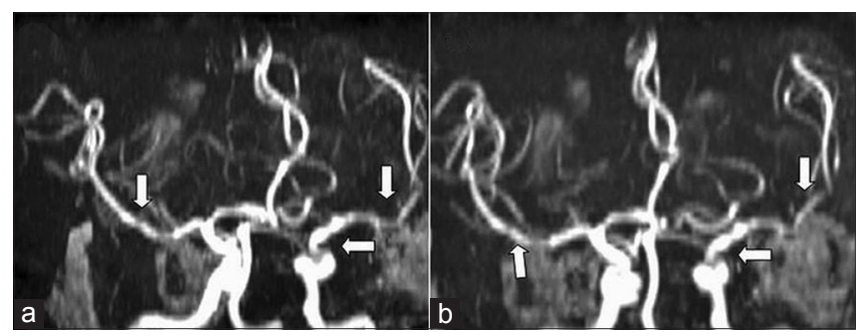

Figure 2: Magnetic resonance angiography of the brain ( $a$ and b) showing irregularity and dilation of intracranial vessels (arrows) 
to the treatment with acyclovir, short course of steroid, anticoagulants, and later antiplatelets. This case report also supports the possibility of contiguous vascular, or CSF or hematogenous spread of the virus in the pathogenesis of zoster-related vasculopathy in addition to the classical theory of neurogenic spread.

Infection with VZV is associated with spectrum of neurological manifestations in humans like zoster, preherpetic and postherpetic neuralgia, myelitis, large vessel granulomatous arteritis, and small vessel encephalitis. ${ }^{[1]}$ Large vessel infarcts following zoster infection of the trigeminal ganglion are thought to be from axonal transport of the virus from trigeminal afferent fibers that innervate the vessels of the anterior cerebral circulation with subsequent vasculitis. ${ }^{[2]}$ There are few reports of cerebral infarcts following sacral and thoracic ganglion involvement, suggesting CSF/hematogenous spread of the virus or simultaneous reactivation in the trigeminal ganglion. ${ }^{[2,3]}$ Recently there have been reports of HIV seropositive and negative patients with bilateral cerebral infarcts with unilateral zoster suggesting vascular spread of the virus, which is similar to our case. ${ }^{[4,6]}$ They also demonstrated, like in our case, the VZV antibodies in CSF and improvement in clinical condition with acyclovir.

Several potential factors contribute to an increased frequency of infarctions in AIDS patients. The best recognized are the infectious complications, which include VZV, cytomegalovirus, tuberculosis, cryptococcosis, syphilis, and toxoplasmosis. ${ }^{[6]}$ We have ruled out conventional causes of stroke and above etiologies including tuberculosis by negative CSF antibodies, other blood and imaging studies. The temporal association of stroke with zoster ophthalmicus, positive CSF antibodies, response to treatment, and absence of other demonstrable etiologies suggest that $\mathrm{VZV}$ is the reason for stroke in our patient. Five modes of VZV infection of the central nervous system in AIDS patients are described: (i) Multifocal encephalitis predominantly involving the white matter likely to be due to hematogenous spread, (ii) ventriculitis due to necrosis of the ventricular wall, (iii) acute hemorrhagic meningo-myeloradiculitis with necrotizing vasculitis due to secondary seeding of the CSF, (iv) focal necrotizing myelitis due to neural spread from the diseased dorsal root ganglion, and (v) vasculopathy involving leptomeningeal arteries and causing cerebral infarcts associated with meningitis. ${ }^{[7]}$

Vasculopathy involving large vessels present with acute stroke with in a mean period of 7 weeks between the onset of neurological disease and zoster, but intervals as long as 6 months have been recorded. Stroke results from bland or hemorrhagic infarcts. Mental symptoms are common and up to $25 \%$ of patients may succumb due to their illness. ${ }^{[8]}$ Cerebral angiography usually shows the focal constriction and segmental narrowing of the affected vessels and commonly middle, anterior, and internal carotid arteries are involved. Other vasculopathies can produce the same neurologic symptoms, signs, CSF changes, and imaging abnormalities; hence detection of anti-VZV IgG antibody in CSF, which is the virologic test of choice is required for the diagnosis. Our patient had presented 3 months after the zoster and had involvement of vessels bilateral to the rash with hemiparesis and encephalopathic symptoms. These features probably suggest that the VZV virus had spread through cerebral arteries or CSF or hematogenous route as compared with classical the trigeminal nerve spread.

Steroids, intravenous acyclovir and anticoagulation (to prevent progression of thrombosis) have been advocated as the treatment modalities. ${ }^{[8]}$ Because the virus is present in the arteries and it is associated with inflammation there is a role for acyclovir and steroids. ${ }^{[2,8,9]}$ We treated our patient with the above modalities and he made a good recovery and was continued on antiplatelet medicine because we could not repeat imaging of the blood vessels. When a diagnosis (clinically and radiologically) of VZV with stroke is suspected, virological confirmation is required. It can be either from detecting VZV DNA by polymerase chain reaction (PCR) or by anti-VZV IgG antibody in CSF. In a study, $93 \%$ of the 30 patients with VZV vasculopathy had anti-VZV IgG in the CSF compared with only $30 \%$ with VZV DNA in CSF by PCR..$^{[1,9]}$ This may be due to protracted clinical course of VZV vasculopathy, usually lasting for weeks to months; hence antigen may disappear and only antibodies might persist. Positive CSF PCR for VZV is helpful; however a negative PCR does not exclude the diagnosis. The HIV itself is a neurotropic virus and has predilection for the endothelial cells and may present with granulomatous angitis, eosinophilic vasculitis, and nonspecific small vessel angitis. The causes of infarctions in AIDS patients include vasculitis, coagulation abnormalities due to virus, infections, neoplasms and cardiac. Viral load has no correlation with vasculopathy and it is a diagnosis of exclusion and it is important that treatable causes are excluded. In our patient it is difficult to exclude the HIV associated primary vasculopathy but clinical circumstances, CSF VZV antibody positivity and response to treatment favor VZV vasculopathy. ${ }^{[10,11]}$

In conclusion, this case report of unilateral zoster ophthalmicus in an immunocompromised patient presenting with bilateral stroke highlights the 
CSF/hematogenous/vascular spread of the virus as opposed to the conventional neurogenic spread. Patient made a good recovery with a choice of modalities of treatment, which act on the various aspects of the pathogenic mechanism of the stroke in a patient with zoster.

\section{References}

1. Gilden DH, Kleinschmidt-DeMasters BK, LaGuardia JJ, Mahalingam R, Cohrs RJ. Neurologic complications of the reactivation of Varicella-Zoster virus. N Engl J Med 2000;342:635-45.

2. Gilden DH, Lipton HL, Wolf JS, Akenbrandt W, Smith JE, Mahalingam R, et al. Two patients with unusual forms of varicella-zoster virus vasculopathy. N Engl J Med 2002;347:1500-3.

3. Rawlinson WD, Cunningham AL. Contralateral hemiplegia following thoracic herpes zoster. Med J Aust 1991;155:344-6.

4. Takeshi S, Chie S, Hiroo W, Masahiro A, Hajime G. Evidence for vascular spread of varicella zoster-associated vasculopathy. Ann Intern Med 2006;144:535-7.

5. Hashemi N, Zhang J, Volpi J, Lee AG, Gordon LK. A pox upon your house. Surv Ophthalmol 2013;58:640-3.
6. Gillams AR, Allen E, Hrieb K, Venna N, Craven D, Carter AP. Cerebral infarction in patients with AIDS. AJNR Am J Neuroradiol 1997;18:1581-5.

7. Gray F, Bélec L, Lescs MC, Chrétien F, Ciardi A, Hassine D, et al. Varicella-zoster virus infection of the central nervous system in the acquired immune deficiency syndrome. Brain 1994;117:987-99.

8. Hilt DC, Buchholz D, Krumholz A, Weiss H, Wolinsky JS. Herpes zoster ophthalmicus and delayed contralateral hemiparesis caused by cerebral angitis: Diagnosis and management approaches. Ann Neurol 1983;14:543-53.

9. Nagel MA, Cohrs RJ, Mahalingam R, Wellish MC, Forghani B, Schiller A, et al. The varicella zoster virus vasculopathies: Clinical, CSF, imaging, and virologic features. Neurology 2008;70:853-60.

10. Connor MD, Lammie GA, Bell JE, Warlow CP, Simmonds P, Brettle RD. Cerebral infarction in adult AIDS patients: Observations from the Edinburgh HIV autopsy cohort. Stroke 2000;31:2117-26.

11. Saravanan M, Turnbull W. Brain: Non-infective and non-neoplastic manifestations of HIV. Br J Radiol 2009;82:956-65.

How to cite this article: Kulkarni GB, Mustare V, Mirza MA. Unilateral herpes zoster ophthalmicus with bilateral cerebral infarcts in human immunodeficiency virus seropositive patient. J Neurosci Rural Pract 2014;5:265-8.

Source of Support: Nil. Conflict of Interest: None declared.

\section{Staying in touch with the journal}

1) Table of Contents (TOC) email alert Receive an email alert containing the TOC when a new complete issue of the journal is made available online. To register for TOC alerts go to www.ruralneuropractice.com/signup.asp.

\section{2) RSS feeds}

Really Simple Syndication (RSS) helps you to get alerts on new publication right on your desktop without going to the journal's website. You need a software (e.g. RSSReader, Feed Demon, FeedReader, My Yahoo!, NewsGator and NewzCrawler) to get advantage of this tool. RSS feeds can also be read through FireFox or Microsoft Outlook 2007. Once any of these small (and mostly free) software is installed, add www.ruralneuropractice.com/rssfeed.asp as one of the feeds. 